\title{
A Comprehensive In Vitro Comparison of Mechanical Properties of Two Rotary Endodontic Instruments
}

\author{
Marco Seracchiani ${ }^{1}$, Gabriele Miccoli ${ }^{2}$, Rodolfo Reda ${ }^{3}$, Alessio Zanza ${ }^{4}$, Federico Valenti Obino ${ }^{5}$, Shilpa Bhandi ${ }^{6}$, \\ Gianluca Gambarini ${ }^{7}$, Luca Testarelli ${ }^{8}$
}

\begin{abstract}
Aim: The aim of the present study was to compare two different nickel-titanium (NiTi) rotary files, F-One Blue and F2 Protaper Gold (PTG), evaluating their properties such as cyclic fatigue resistance, torsional resistance, and bending properties.

Materials and methods: Sixty F-One Blue (25.06) and 60 PTGF2 have been randomly divided into 3 groups of 20 instruments each. Each group was tested in a different way, in order to analyze three different properties: cyclic fatigue resistance, torsional resistance, and flexibility. Cyclic fatigue resistance was evaluated by inserting the instruments for $16 \mathrm{~mm}$ in a stainless-steel artificial canal with a $90^{\circ}$ angle and $5 \mathrm{~mm}$ of curvature with recommended speed and torque. Time to fracture was recorded using a chronometer (1/100 seconds). The number of cycles to fracture (NCF) was then calculated. Fragments were collected, and their lengths were measured. Torsional resistance was evaluated on the apical $3 \mathrm{~mm}$ of each of the files to calculate torque to fracture (TtF) using an endodontic motor (KaVo, Biberach, Germany), which automatically recorded the torque values every $1 / 10 \mathrm{~s}$. All instruments were rotated at the same speed ( $300 \mathrm{rpm}$ ) and torque value (5.5 Ncm). Flexibility was evaluated using a calibrated load cell supported by a computer program. All the collected data were statistically analyzed ( $t$-test) with a significance level set at $5 \%$. Results: A significant difference in terms of cyclic fatigue resistance, torsional resistance, and flexibility between F-One Blue and F2 PTG was found $(p<0.05$ ). The mean value of NCF was 604.16 (SD \pm 23.32 ) for F-One Blue and 300.5 (SD 19.92) for F2 PTG. The mean value of TtF was $1.41 \mathrm{Ncm}(\mathrm{SD} \pm 0.01)$ and $1.39 \mathrm{Ncm}(\mathrm{SD} \pm 0.01)$ for F2 PTG. The mean value of bending test was $29 \mathrm{gcm}(\mathrm{SD} \pm 1.15)$ for F-One Blue and $50 \mathrm{gcm}$ (SD \pm 2.30$)$ for F2 PTG.

Conclusion: F-One Blue better resists to flexural and torsional stresses and seemed to be more flexible. Since F-One Blue mechanical tested performances were better than the F2 PTG ones, these instruments should be considered a very promising instrument.

Clinical significance: As evidenced by the results of this study, F-One Blue should be considered a very promising instrument that could improve endodontic clinical practice.

Keywords: Clinical use, Endodontic, F-One blue, NiTi files, Protaper gold.

World Journal of Dentistry (2020): 10.5005/jp-journals-10015-1729
\end{abstract}

\section{INTRODUCTION}

The introduction of nickel-titanium (NiTi) in the last decades has progressively improved the simplicity, user-friendliness, and the predictability of the root canal treatment $(\mathrm{RCT})$, by reducing number of instruments and operative times. Despite many advantages, intracanal separation of rotary NiTi instruments, it is still a rather frequent iatrogenic error that may negatively affect the outcome of the treatment. ${ }^{1,2}$ The intracanal separation is usually a sudden, unexpected accident because it is not anticipated by any kind of macroscopic deformation or cracks.

Instrumentation stresses are related to the complexity of root canal anatomy. Using cone beam computed tomography (CBCT), it is possible to better evaluate endodontic anatomy in threedimensions and to correctly visualize canal trajectories, avoiding superimposition of hard tissues., ${ }^{3,4}$ Thanks to this technology is possible to identify, during the clinical practice, hidden curvatures, confluent or diverging canals, and bifurcation that are among the most stressful conditions for the NiTi rotary instruments. Unfortunately, CBCT on a routine basis is nowadays used only by a minority of clinicians due to increased costs and radiation risk.

Since canal anatomy is complex and cannot be modified, the current best way to decrease the percentage of intracanal separation, besides using safer motions during the instrumentation, ${ }^{5,6}$ is to improve the clinical performance of the new NiTi instruments. This is currently possible thanks to the several studies investigating
$1-5,7,8$ Department of Oral and Maxillo-Facial Sciences, Sapienza University of Rome, Rome, Italy

${ }^{6}$ Department of Restorative Dental Sciences, Jazan University, Kingdom of Saudi Arabia

Corresponding Author: Alessio Zanza, Department of Oral and Maxillo-Facial Sciences, Sapienza University of Rome, Rome, Italy, Phone: +39 3348731467, e-mail: ale.zanza@gmail.com

How to cite this article: Seracchiani M, Miccoli G, Reda R, et al. A Comprehensive In Vitro Comparison of Mechanical Properties of Two Rotary Endodontic Instruments. World J Dent 2020;11(3):185-188.

Source of support: Nil

Conflict of interest: None

metallurgical properties of $\mathrm{NiTi}$ and the mechanical properties of the NiTi instruments. ${ }^{7-9}$ The most common studies available in the literature are in vitro evaluations and comparisons of mechanical properties of the NiTi instruments. These studies investigated mainly the following properties: flexibility, cutting efficiency, cyclic fatigue, and torsional resistance. Cyclic fatigue and torsional resistance are physical features of a NiTi instrument. The first one is the ability to resist at repeated cycles of tension and compression in a curved canal and it is an evaluation factor of flexural strength during bending, while the second one is the instrument's ability to be not damaged when the tip becomes wedged in the canal 
and the shaft continues to rotate. Thus, flexibility and torsional resistance are important properties that could affect the mechanical performance of NiTi files during the root canal preparation, and they are influenced by several factors. ${ }^{10-15}$ Among these factors, two were found to be most relevant in determining resistance to bending and torsional stress: the heat treatment and the mass of the NiTi instruments. The heat treatment modifies the instrument behavior simply changing the transition temperature of the instrument. ${ }^{16,17}$ A more martensitic file will be more resistant to flexural stress, while a more austenitic instrument will be more resistant to torsional fatigue. Moreover, the mass at the point of maximum stress is a key factor for the resistance of the instrument. Higher the mass, higher the torsional resistance will be, but on the contrary, it will be less resistant to flexural failure. ${ }^{18,19}$

Unfortunately, improving a specific property of an instrument may result in decreasing other properties. Therefore, when analyzing and comparing instruments in vitro, many or ideal all properties should be investigated.

The aim of the present study was to compare the different properties (cyclic fatigue resistance, torsional resistance, and bending properties) of two different NiTi rotary files, F-One Blue, an instrument recently introduced in the market, and F2 PTG, a well-studied and worldwide spread rotary file.

\section{Materials and Methods}

All steps of this study were performed at the Department of Oral and Maxillo-Facial Sciences of Sapienza University of Rome (Rome, Italy). Sixty F-One Blue 25.06 (Fanta Dental, Shanghai, China) and 60 PTG F2 (Dentsply Maillefer, Ballaigues, Switzerland) have randomly divided into 3 groups of 20 instruments each. Three groups have been randomly selected to be tested in three different ways: cyclic fatigue resistance, torsional resistance, and flexibility. Before undergoing the above-mentioned tests, all the 120 rotary files were previously examined through stereomicroscope (Carl Zeiss Micro Imaging, Göttingen, Germany) at 20× magnification to detect any macroscopic defects. No rotary files were discarded due to macroscopic external defects. The same operator performed all the tests, to avoid differences due to operator skills.

\section{Cyclic Fatigue Test}

Twenty F-One Blue and 20 F2 PTG were tested using a cyclic fatigue device already validated in the previous study, made of two main parts: a stainless steel artificial (SS) canal with a $90^{\circ}$ angle and $5 \mathrm{~mm}$ of curvature and a hand piece connected to a mobile device which allowed easy repeatable insertion of the instrument in the abovementioned SS root canal. ${ }^{20-23}$ The NiTi rotary files were inserted for $16 \mathrm{~mm}$ inside the canal and rotated with the speed and the torque recommended by the manufacturer, $500 \mathrm{rpm}$ and $2 \mathrm{Ncm}$ for F-One Blue and $300 \mathrm{rpm} 2.5 \mathrm{Ncm}$ for PTG F2. The time to fracture was recorded using a chronometer (1/100 seconds), and the time was stopped when a visible or audible sign of fracture was detected. Finally, the NCF was calculated by multiplying the time to fracture for the rotational speed. Fragments were collected and their lengths were measured using a digital caliber.

\section{Torsional Resistance Test}

A torsional test was performed on the apical $3 \mathrm{~mm}$ of each of the files to calculate TtF using a new device validated in recently published manuscripts. ${ }^{11}$ This device consists of two main parts, a hand piece connected on one side to an electric motor (KaVo,
Biberach, Germany) allowing a real-time (0.1 seconds) recording of the torque with a sensitivity of $0.05 \mathrm{Ncm}$ and a vise used to firmly secure the apical $3 \mathrm{~mm}$ of the NiTi instruments. The two abovementioned machines were linked using a mobile device allowing easy placement of the files inside the vise. All the instruments were rotated at the same speed $300 \mathrm{rpm}$. The torque value set on the motor was $5.5 \mathrm{Ncm}$, to ensure a precise recording in a range from 0.05 to $5.5 \mathrm{Ncm}$. The TtF was registered for each instrument by the integrated software of the hand piece and calculated using a spreadsheet.

\section{Bending Test}

The bending test was performed by the using of a custom-made calibrated load cell and was supported by a computer program that guarantees a correct and reliable recording value expressed in $\mathrm{gcm}$. The tip of the instrument was locked for $3 \mathrm{~mm}$ with a vise to the calibrated load cell. Once the instrument underwent an elastic displacement and reached an excursion of $45^{\circ}$, the moment of bending was recorded. ${ }^{24}$ This test was carried out for all the tested instruments.

\section{Statistical Analysis}

All the collected data were entirely analyzed using the Statistical Package for the Social Sciences (IBM-SPSS, version 23, Shanghai, China). A $t$-test was performed for cyclic fatigue, torsional resistance, and flexibility tests, $p$ values were set at 0.05 .

\section{Results}

Table 1 shows the results of cyclic fatigue tests, reporting mean values and standard deviation of NCF and fragment lengths (FLs). F-One Blue showed statistically significant higher values of NCF than PTG F2 $(p<0.05)$.

Table 2 shows the results of the torsional resistance; the F-One Blue showed significantly higher resistance $(p<0.05)$ when compared to the PTG F2.

Table 2 shows the results of bending test. The F-One Blue has significantly lower resistance $(p<0.05)$ when compared to the PTG F2, which stands for a greater flexibility.

\section{Discussion}

The increased production and the spread of the NiTi rotary instruments among not only endodontist but also general practitioners (GP) is due to improved properties and a hugely increased number of new instruments recently introduced on the market. The multitude of new instruments available on the market can generate confusion, mainly because manufacturers tend to highlight the improvement of some properties and do the opposite in the case of a decrease of other properties related to innovations. The same procedure is sometimes done by researchers, investigating only one property of the new instrument. For these reasons, when a new instrument is introduced on the market, a comprehensive, wide evaluation of all the mechanical properties should be performed. Despite the lack of standardized dynamic test, the most common static tests, although not exhaustive, must be used to evaluate and compare new instruments.

The PTG F2 is a deeply studied instrument with a convex triangular cross-section, with 3 cutting surfaces along the file surface. It presents a tip of 25 and a variable taper. The heat-treated alloy of PTG F2 instruments is the Gold Wire (Dentsply Maillefer, 
Table 1: Mean and $p$ values for NCF and FLs of F-One Blue and PTG F2

\begin{tabular}{llllll}
\hline & \multicolumn{2}{c}{ NCF } & & \multicolumn{2}{c}{ FLs } \\
\cline { 2 - 3 } \cline { 5 - 6 } & F-One Blue & PTG F2 & & F-One Blue & PTG F2 \\
\hline Mean \pm SD & $604.16 \pm 23.32$ & $300.5 \pm 19.92$ & & $6.05 \pm 0.30$ & $6.075 \pm 0.01$ \\
$p$ value & 0.000 & & 0.85 & \\
\hline
\end{tabular}

Table 2: Mean and $p$ values for TtF and bending test of F-One Blue and PTG F2

\begin{tabular}{llllll}
\hline & \multicolumn{2}{c}{ TtF } & & \multicolumn{2}{c}{ Bending } \\
\cline { 2 - 3 } \cline { 5 - 6 } & F-One Blue & PTG F2 & & F-One Blue & PTG F2 \\
\hline Mean \pm SD & $1.41 \pm 0.01$ & $1.39 \pm 0.01$ & & $29 \pm 1.15$ & $50 \pm 2.30$ \\
$p$ value & 0.002 & & & 0.000 & \\
\hline
\end{tabular}

Ballaigues, Switzerland) that according to the previous studies increases their fatigue resistance and flexibility. ${ }^{16,17}$

The F-One Blue rotary instruments are recently introduced on the market with a peculiar flat-side design along the long axis of the instrument. ${ }^{23}$ This instrument is designed to be used as a single-file system, with a tip size 25 and a 0.06 constant taper. It exhibits an S-shaped cross section modified by the flat design. Another innovative characteristic is the proprietary, (not disclosed) heat treatment, the AF-H wire (Fanta Dental, Shanghai, China). This heat treatment, according to the manufacturer internal studies, increased both torsional and flexural resistance, but no data are currently available in the dental literature.

The results from the present study showed higher cyclic fatigue resistance, torsional resistance, and an increased flexibility of the F-One Blue, when compared with the PTG F2. Cyclic fatigue and torsional resistances are influenced by several factors, among them the most remarkable are heat treatment, metal mass, and cross-sectional design. . $^{4,15,20,21,25,26}$ No published studies analyzed or compared the differences between the two proprietary heat treatments; therefore, their influence in the present results is a possibility but cannot be demonstrated.

The cross-sectional design and the relative metal mass could be taken into consideration to explain the better result of F-One Blue in flexural resistance. The F-One Blue presents a flat-sided S-shaped cross-sectional design along the surface of the instrument. The combination of this flat design and the S-shaped cross-sectional design gives the instrument a lower metal mass. ${ }^{18,23,27}$ In literature, many studies evaluated the role of the metal mass on cyclic fatigue failure and have already demonstrated that the lower the mass, the greater the resistance to cyclic fatigue will. These features can also explain the results of the flexibility test; the less the mass, the more flexible the instrument is.

The results of the torsional test showed a higher torque at fracture (greater torsional resistance) of F-One Blue compared to PTG F2. This was an unexpected result, because it has been widely demonstrated that smaller dimensions and (or smaller metal mass significantly decrease torsional resistance. ${ }^{28-30}$ These unexpected results can be explained by the new manufactured heat-treated alloy, the AF-H wire. According to the manufacturer internal studies, this peculiar heat treatment was able to increase the metallurgical properties, also providing a superior resistance to torsional stresses.

In conclusion, within the limitations of this study and the lack of clinical studies comparing the in vivo performance of the tested $\mathrm{NiTi}$ rotary files, these results showed significant differences in the in vitro performance for F-One Blue compared to PTG F2. Since the PTG F2 is a commonly used and widely investigated instrument, it could be stated that the new F-One Blue can be considered a very promising instrument, due to its innovative flat side design and heat treatment, and its clinical use can be promoted.

\section{References}

1. Kottoor J, Velmurugan N, Gopikrishna V, et al. Effects of multiple root canal usage on the surface topography and fracture of two different $\mathrm{Ni}-\mathrm{Ti}$ rotary file systems. Indian J Dent Res 2013;24(1):42-47. DOI: 10.4103/0970-9290.114942.

2. Gambarini G, Miccoli G, Seracchiani M, et al. Fatigue resistance of new and used nickel-titanium rotary instruments: a comparative study. Clin Ter 2018;169(3):e96-e101. DOI: 10.7417/T.2018.2061.

3. Segato AVK, Piasecki L, Felipe Iparraguirre Nuñovero M, et al. The accuracy of a new cone-beam computed tomographic software in the preoperative working length determination ex vivo. J Endod 2018;44(6):1024-1029. DOI: 10.1016/j.joen.2018.02.027.

4. Valenti-Obino F, Di Nardo D, Quero L, et al. Symmetry of root and root canal morphology of mandibular incisors: a cone-beam computed tomography study. J Clin Exp Dent 2019;11(6):e527-e533. DOI: 10.4317/jced.55629.

5. Gambarini G, Tucci E, Bedini R, et al. The effect of brushing motion on the cyclic fatigue of rotary nickel titanium instruments. Ann Ist Super Sanita 2010;46(4):400-404. DOI: 10.4415/ANN_10_04_07.

6. Gambarini G, Piasecki L, Miccoli G, et al. Classification and cyclic fatigue evaluation of new kinematics for endodontic instruments. Aust Endod J 2019;45(2):154-162. DOI: 10.1111/aej.12294.

7. Necchi S, Petrini L, Taschieri S, et al. A comparative computational analysis of the mechanical behavior of two nickel-titanium rotary endodontic instruments. J Endod 2010;36(8):1380-1384. DOI: 10.1016/j.joen.2010.03.026.

8. Alcalde MP, Tanomaru-Filho M, Bramante CM, et al. Cyclic and torsional fatigue resistance of reciprocating single files manufactured by different nickel-titanium alloys. J Endod 2017;43(7):1186-1191. DOI: 10.1016/j.joen.2017.03.008.

9. Yang YJ, Hou BX, Hou XM. Metallurgic behavior and mechanical property of nickel-titanium endodontic files made by 3 heat treatment techniques. Zhonghua Kou Qiang Yi Xue Za Zhi 2018;53(8):539-545. DOI: 10.3760/cma.j.issn.1002-0098.2018.08.008.

10. Plotino G, Giansiracusa Rubini A, Grande NM, et al. Cutting efficiency of reciproc and WaveOne reciprocating instruments. J Endod 2014;40(8):1228-1230. DOI: 10.1016/j.joen.2014.01.041.

11. Gambarini G, Galli M, Seracchiani M, et al. In vivo evaluation of operative torque generated by two nickel-titanium rotary instruments during root canal preparation. Eur J Dent 2019;13(4): 556-562. DOI: 10.1055/s-0039-1698369.

12. Gambarini G, Seracchiani M, Piasecki L, et al. Measurement of torque generated during intracanal instrumentation in vivo. Int Endod J 2019;52(5):737-745. DOI: 10.1111/iej.13042.

13. Gambarini G, Galli M, Di Nardo D, et al. Differences in cyclic fatigue lifespan between two different heat treated NiTi endodontic 
rotary instruments: WaveOne gold vs EdgeOne fire. J Clin Exp Dent 2019;11(7):e609-e613. DOI: 10.4317/jced.55839.

14. Gambarini G, Miccoli G, D'Angelo M, et al. The relevance of operative torque and torsional resistance of nickel-titanium rotary instruments: a preliminary clinical investigation. Saudi Endod J 2020. in press.

15. Di Nardo D, Gambarini G, Seracchiani M, et al. Influence of different cross-section on cyclic fatigue resistance of two nickel-titanium rotary instruments with same heat treatment: an in vitro study. Saudi Endod J 2020. in press.

16. Ebihara A, Yahata Y, Miyara K, et al. Heat treatment of nickel-titanium rotary endodontic instruments: Effects on bending properties and shaping abilities. Int Endod J 2011;44(9):843-849. DOI: 10.1111/j.13652591.2011.01891.x

17. Miccoli G, Gaimari G, Seracchiani M, et al. Resistance to fracture of two nickel-titanium rotary instruments made with different thermal treatments. Ann Stomatol (Roma) 2017;8(2):53-58. DOI: 10.11138/ ads/2017.8.2.059.

18. Basheer Ahamed SB, Vanajassun PP, Rajkumar K, et al. Comparative evaluation of stress distribution in experimentally designed nickeltitanium rotary files with varying cross sections: a finite element analysis. J Endod 2018;44(4):654-658. DOI: 10.1016/j.joen.2017. 12.013.

19. Pedullà E, Plotino G, Scibilia M, et al. Cyclic fatigue comparison among endodontic instruments with similar cross section and different surface coating. Minerva Stomatol 2019;68(2):67-73. DOI: 10.23736/ S0026-4970.17.04009-2.

20. Grande NM, Plotino G, Pecci R, et al. Cyclic fatigue resistance and three-dimensional analysis of instruments from two nickel-titanium rotary systems. Int Endod J 2006;39(10):755-763. DOI: 10.1111/j.13652591.2006.01143.x.

21. Plotino G, Grande NM, Cordaro M, et al. Measurement of the trajectory of different NiTi rotary instruments in an artificial canal specifically designed for cyclic fatigue tests. Oral Surg Oral Med
Oral Pathol Oral Radiol Endod 2009;108(3):e152-e156. DOI: 10.1016/ j.tripleo.2009.05.046.

22. Pedullà E, La Rosa GRM, Virgillito $C$, et al. Cyclic fatigue resistance of nickel-titanium rotary instruments according to the angle of file access and radius of root canal. J Endod 2020;46(3):431-436. DOI: 10.1016/j.joen.2019.11.015.

23. Gambarini G, Miccoli G, Seracchiani M, et al. Role of the flat-designed surface in improving the cyclic fatigue resistance of endodontic $\mathrm{NiTi}$ rotary instruments. Materials (Basel) 2019;12(16):2523.

24. Özyürek T, Gündoğar M, Yılmaz K, et al. Bending resistance and cyclic fatigue life of reciproc blue, WaveOne gold, and genius files in a double (S-shaped) curved canal. J Dent Res Dent Clin Dent Prospects 2017;11(4):241-246. DOI: 10.15171/joddd.2017.042.

25. Modesto TC, Acosta ECP, Resende PD, et al. Cyclic flexural fatigue resistance of NiTicontrolled memory and blue technology instruments after torsional preloading. J Appl Oral Sci 2018;26(0):e20180144. DOI: 10.1590/1678-7757-2018-0144.

26. Özyürek T, Uslu G, Gündoğar M, et al. Comparison of cyclic fatigue resistance and bending properties of two reciprocating nickeltitanium glide path files. Int Endod J 2018;51(9):1047-1052. DOI: 10.1111/iej.12911.

27. De-Deus G, Leal Vieira VT, Nogueira da Silva EJ, et al. Bending resistance and dynamic and static cyclic fatigue life of reciproc and WaveOne large instruments. J Endod 2014;40(4):575-579. DOI: 10.1016/j.joen.2013.10.013.

28. Campbell L, Shen Y, Zhou HM, et al. Effect of fatigue on torsional failure of nickel-titanium controlled memory instruments. J Endod 2014;40(4):562-565. DOI: 10.1016/j.joen.2013.12.035.

29. Di Nardo D, Galli M, Morese A, et al. A comparative study of mechanical resistance of two reciprocating files. J Clin Exp Dent 2019;11(3):e231-e235. DOI: 10.4317/jced.55487.

30. Alqedairi A, Alfawaz $\mathrm{H}$, Abualjadayel $\mathrm{B}$, et al. Torsional resistance of three Protaper rotary systems. BMC Oral Health 2019;19(1):124. DOI: 10.1186/s12903-019-0820-7. 